\title{
Spontaneous intracranial hypotension associated with posterior reversible encephalopathy syndrome
}

\author{
Subham Choudhary ${ }^{1}$, Kanika Sekhri Sethi ${ }^{2}$, Satyan Nanda ${ }^{3}$, Prem Kumar Ganesan ${ }^{2}$, \\ Man Mohan Mehndiratta ${ }^{3}$, Rajiv Anand ${ }^{3}$, Rahul Handa ${ }^{3}$

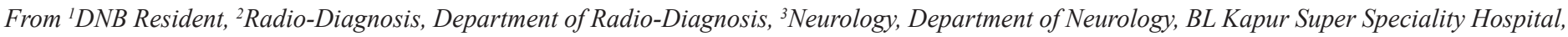 \\ New Delhi, Delhi, India
}

\begin{abstract}
Spontaneous intracranial hypotension (SIH) is usually due to cerebrospinal fluid (CSF) leak, resulting in loss of CSF volume. Posterior reversible encephalopathy syndrome (PRES) is the inability of the posterior circulation of the brain to autoregulate in response to significant variation in blood pressure. Altered perfusion with associated blood-brain barrier disruption may lead to vasogenic edema, usually without infarction, commonly in the parieto-occipital regions. Magnetic resonance (MR) imaging of the brain and spine and cerebral MR venography are essential tools for diagnosing clinically suspected SIH and PRES. We present 18-year-young gentleman with clinical and radiological signs of SIH, which later evolved toward PRES.
\end{abstract}

Key words: Cerebrospinal fluid, Magnetic resonance imaging, Posterior reversible encephalopathy syndrome, Spontaneous intracranial hypotension

$\mathrm{S}$ pontaneous intracranial hypotension $(\mathrm{SIH})$ is an uncommon disease of low cerebrospinal fluid (CSF) pressure as a result of CSF volume depletion or increased absorption. No evident gender prevalence has been noted; however, there is female preponderance [1]. Posterior reversible encephalopathy syndrome (PRES) is a neurological condition that occurs due to the inability of the brain circulation to autoregulate in response to sudden changes in blood pressure. The radiological and clinical alterations are completely reversible after treatment [2]. Pathogenesis of PRES has been linked to both arterial and venous cerebrovascular dysfunction. The most of the patients with PRES have markedly raised blood pressure [3]. PRES is also seen in organ transplantation, severe infections, sepsis, shock, and autoimmune diseases [4].

We present a case of SIH associated with PRES in an 18-year-young gentleman who presented with the signs of SIH and later developed signs of PRES during his course in the hospital.

\section{CASE REPORT}

An 18-year-old male presented to the emergency department of our institute with complaints of acute onset, gradually progressive headache for the past 1 week which was associated with recurrent

\section{Access this article online}

Received - 29 December 2021

Initial Review - 07 January 2022

Accepted - 24 January 2022

DOI: $110.32677 /$ ijcr.v8i1.3211 bouts of projectile vomiting approximately 2-3 times a day. Headache was episodic, mainly in the occipital region, severe in intensity with throbbing nature, worsens on assuming an upright posture, and improved completely in a recumbent position. The medical history was significant for occasional headaches with vomiting and photophobia. These used to be infrequent events, usually hemicranial, lasting for 4-6 h with no postural variation, and relieved with analgesic and rest.

His blood pressure and pulse rate were well within normal limits. General examination was suggestive of marfanoid habitus (arm span to height: 1.05). The neurological examination was normal except for mild neck rigidity. Because of strong postural relation to headache, occipital location, and marfanoid habitus, a clinical possibility of headache secondary to intracranial hypotension was thought.

The patient was subjected to plain and contrast-enhanced magnetic resonance imaging (MRI) and MR venography (MRV) of the brain and MRI of the spine to confirm the diagnosis and etiology of headache. MRI brain revealed swollen dural venous sinuses, superior convex margin with the homogeneously enhancing pituitary gland, and decreased mamillo-pontine angle. No parenchymal lesion or subdural collection was seen on MRI. MRV ruled out dural venous sinus thrombosis and MR angiography of the circle of Willis was also unremarkable. MRI of the spine revealed spinal longitudinally extradural collection in the dorsal spine posteriorly and the dorsal cord

Correspondence to: Subham Choudhary, Department of Radio-diagnosis, BL Kapur Hospital, New Delhi, India. E-mail: subh.awesome10@gmail.com

(C) 2022 Creative Commons Attribution-NonCommercial 4.0 International License (CC BY-NC-ND 4.0). 
was displaced anteriorly. There was no signal change within the cord. These findings strongly suggested the diagnosis of SIH (Fig. 1). Computed tomography (CT) myelography was done to locate the site of the CSF leak. Opening CSF pressure measured at the time of CT myelography was low $(20 \mathrm{~mm}$ of $\mathrm{Hg}$ ), but it did not demonstrate any CSF leak. CSF collected at the time of myelography revealed elevated protein levels: $129.1 \mathrm{mg} / \mathrm{dL}$ and normal cell count and glucose levels. CSF sent for BioFire and the panel was negative. Gene X-pert for tuberculosis and India ink preparation for Cryptococcus were negative.

On Day 4 of admission, the patient developed a severe headache which was holocranial, worsens on lying down position, and was associated with vomiting. Repeat MRI brain was done, which showed $\mathrm{T} 2 \mathrm{~W}$ and fluid-attenuated inversion recovery hyperintensity involving the right cerebellum, bilateral frontoparietal, bilateral occipital, and the left temporal cortex. Mild restricted diffusion with a mild enhancement of the right cerebellar lesion was noted. The rest of the areas did not show any restricted diffusion. These findings in the context of the given clinical picture were suggestive of PRES (Fig. 2).

The next day he had three episodes of generalized tonic-clonic seizures. This was associated with a markedly elevated blood pressure of $180 / 120 \mathrm{~mm}$ of $\mathrm{Hg}$ and a fall in heart rate (HR) to 42 beats/min. Repeat MRI brain showed a significant increase of edema with involvement of the left cerebellar region and the mass effect was observed on the brainstem and the fourth ventricle with left inferior tonsillar herniation. In the context of the imaging findings, a CT angiogram of the head-and-neck was done, which was unremarkable. A final diagnosis of PRES in view of raised blood pressure and reduced cerebral pressure was considered.
As the patient was not hemodynamically stable and no exact site of CSF leak was known, aggressive lowering of blood pressure control was preferred over epidural blood patch. Electroencephalography showed generalized inter-ictal spike and wave discharges with background slowing. Serum autoimmune/paraneoplastic panel was negative. Autoimmune workup: Antinuclear antibody (ANA), ANA profile, angiotensinconverting enzyme levels, antiphospholipid antibodies, and antineutrophil cytoplasmic antibodies profile were all negative. NMO and anti-MOG antibody profiles were negative. Renal Doppler done in view of accelerated hypertension was normal. With the above-mentioned medical treatment, the patient started showing clinical improvement, and by the time of discharge (Day 14 of admission), he was completely asymptomatic. Follow-up MRI brain was done at the time of discharge and outpatient department follow-up (3 months follow-up) showed continued improvement in imaging findings. He was given levetiracetam and metoprolol for 2 months and is now asymptomatic.

\section{DISCUSSION}

The combination of SIH and PRES has been described in the literature. SIH is a syndrome characterized by postural headaches elicited or exacerbated by upright position, caused by a reduction of CSF volume. Due to non-specific signs and symptoms, SIH is frequently underdiagnosed in daily clinical practice. Cranial MRI findings are also non-specific. MRI of the spine based on T2-weighted image or IV contrast-enhanced T1-weighted image shows dilated epidural and intradural veins, extrathecal CSF collections, dural contrast enhancement, syringomyelia, and fluid
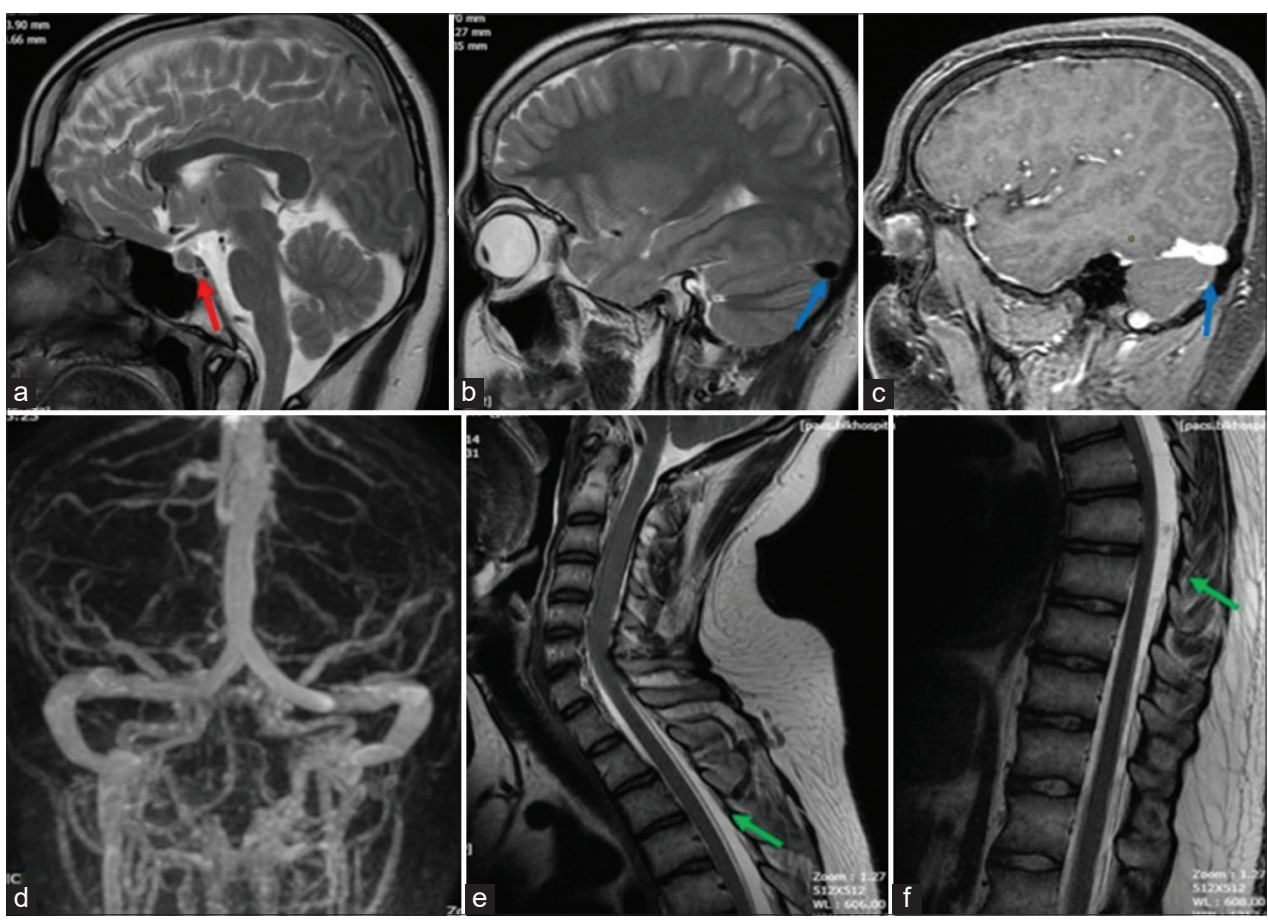

Figure 1: (a) T2W sagittal image shows convex superior margin of pituitary gland (red arrow); (b and c) T2W and post contrast T1W sagittal images show distended transverse sinus (blue arrows); (d) On magnetic resonance venography, no venous thrombosis noted; (e and f) T2W sagittal images of cervico-dorsal spine reveal spinal longitudinal extradural collection in the dorsal spine posteriorly and the dorsal cord was displaced anteriorly (green arrows). Imaging findings favour spontaneous intracranial hypotension 


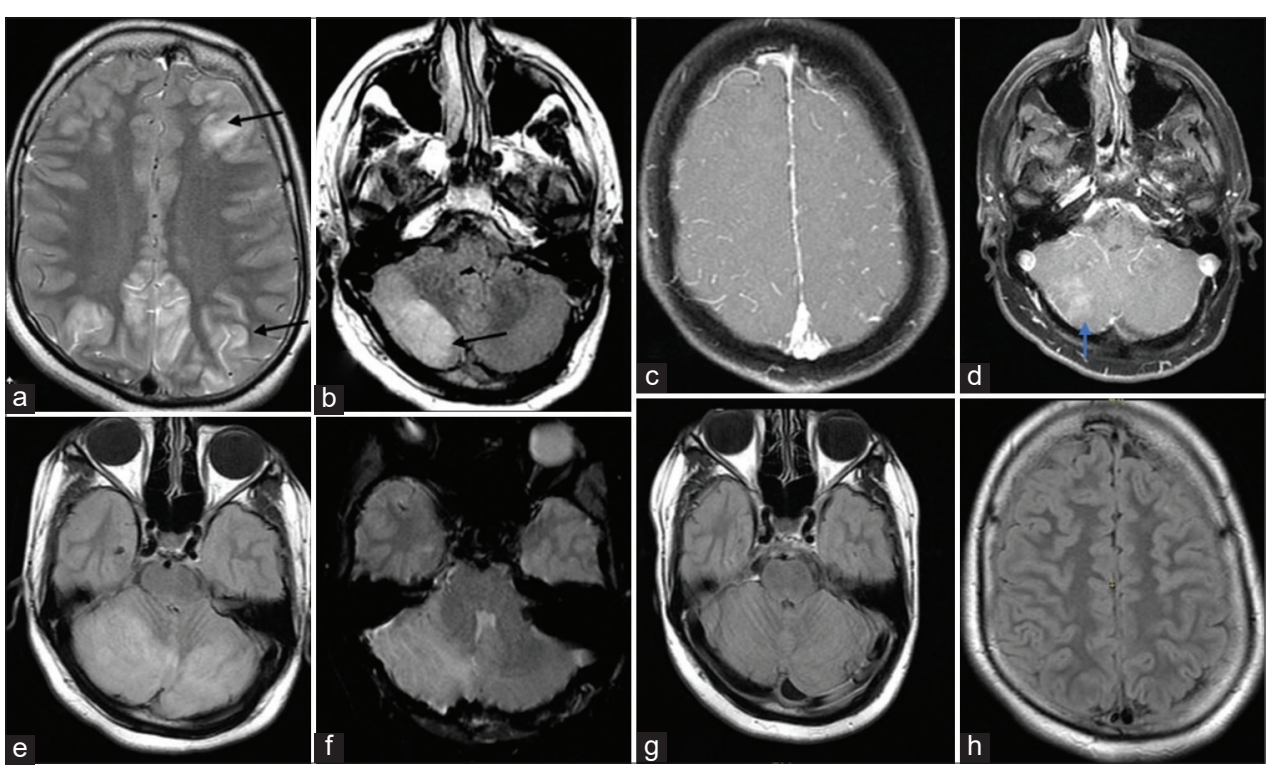

Figure 2: (a and b) Magnetic resonance imaging (MRI) done 5 days after initial MRI: Fluid-attenuated inversion recovery (FLAIR) images revealed patchy areas of hyperintense signal (arrows) in bilateral cerebral hemispheres and right cerebellar hemisphere (arrows) with mild patchy enhancement of the right cerebellar lesion (c and d). Follow-up MR done after 6 days: FLAIR image revealed increase in areas of signal change, now involving bilateral cerebellar hemispheres (e) with no hemorrhage on gradient image (f). MRI done after 5 weeks: FLAIR image revealed resolution of signal ( $g$ and $h)$

Table 1: Different studies related to the association of SIH and PRES and relevant hypothesis explaining them

\begin{tabular}{lll}
\hline Author & Year & Hypothesis to explain the association of SIH and PRES \\
\hline Pugliese et al. [2] & 2010 & $\begin{array}{l}\text { Venous congestion subsequent to venous engorgement } \\
\text { consistent with Monroe-Kelly hypothesis }\end{array}$ \\
Santillan et al. [3] & 2016 & $\begin{array}{l}\text { In response to loss of CSF and intracranial hypotension, } \\
\text { the body may compensate by enhancing cerebral } \\
\text { perfusion pressure. In extreme states, this hyperperfusion } \\
\text { may result in autoregulatory failure leading to PRES }\end{array}$ \\
Savoyard et al. [10] & 2007 & $\begin{array}{l}\text { Explained the impairment in the drainage of the deep } \\
\text { venous system due to functional stenosis of vein of Galen } \\
\text { while entering the straight sinus caused by brain sagging }\end{array}$ \\
\hline
\end{tabular}

SIH: Spontaneous intracranial hypotension, PRES: Posterior reversible encephalopathy syndrome, CSF: Cerebrospinal fluid

collections at cervical level [5]. In our case, the patient's altered status was initially attributed to brain $\mathrm{SIH}$; his further clinical deterioration was more due to PRES. Although rare, cases of intracranial hypotension, and PRES have been previously reported in the literature. This case highlights a more unusual presentation of SIH and PRES secondary to spontaneous CSF leak.

There are no specific criteria for diagnosing PRES and the clinical symptoms are often non-specific [6]. The diagnosis relies mostly on typical MR findings and their disappearance over time [7]. The pathogenesis of PRES is still unclear with three possible hypotheses have been proposed (Table 1), which include (a) cerebral vasoconstriction leading to subsequent infarcts in the brain, (b) cerebral autoregulation failure with vasogenic edema, and (c) damage of endothelium with disruption of blood-brain barrier causing fluid and protein transudation and leakage in the brain [8]. The clinical findings and previous medical history in our patient were also non-specific and were not very helpful in making a diagnosis. The radiological findings in later scans, however, were consistent with PRES.

Several cases suggest that downward sagging of the brain in $\mathrm{SIH}$, along with the release of chemical and neurogenic factors, may lead to transient multifocal vasoconstriction of large and medium-sized arteries, which may further attribute to PRES.

In our patient, SIH was probably spontaneous. The main symptom of SIH was recurrent bouts of projectile vomiting and a throbbing headache. Not many cases confirming the association between SIH and PRES have been reported so far in the literature. Two different mechanisms have been put forward which is involved in IH and can lead to PRES. Monroe-Kelly have explained about the venous stagnation due to venous engorgement [9]. Savoyard et al. have explained the impaired drainage of the deep venous system by functional stenosis of vein of Galen entering the straight sinus caused by brain sagging [10]. In our case, distended dural venous sinuses and decreased mamillopontine angle, which can throw weight behind the hypothetic association between $\mathrm{SIH}$ and PRES.

\section{CONCLUSION}

Data from multiple studies have put forward various hypotheses to suggest a correlation between SIH and PRES and that SIH may lead to PRES or PRES-like clinical and radiological patterns. 
Further studies are required for validation of the hypothesis and to establish a better clinic-radiological correlation.

\section{REFERENCES}

1. Petramfar P, Mohammadi SS, Hosseinzadeh F. Treatment of idiopathic intracranial hypotension with tea: A case report. Iran Red Crescent Med J 2016;18:e24620.

2. Pugliese S, Finocchi V, Borgia ML, Nania C, Della Vella B, Pierallini A, et al. Intracranial hypotension and PRES: Case report. J Headache Pain 2010;11:437-40

3. Santillan A, Aamodt W, Bhavaraju-Sanka R. Pearls \& Oy-sters: Spontaneous intracranial hypotension and posterior reversible encephalopathy syndrome. Neurology 2016;86:e55-7.

4. Hobson EV, Craven I, Blank SC. Posterior reversible encephalopathy syndrome: A truly treatable neurologic illness. Perit Dial Int 2012;32:590-4.

5. Albes G, Weng H, Horvath D, Musahl C, Bäzner H, Henkes H. Detection and treatment of spinal CSF leaks in idiopathic intracranial hypotension. Neuroradiology 2012;54:1367-73.

6. Smets GJ, Loyson T, Van Paesschen W, Demaerel P, Nackaerts K. Posterior reversible encephalopathy syndrome possibly induced by pemetrexed maintenance therapy for lung cancer: A case report and literature review. Acta Clin Belg 2018;73:382-8.

7. Lamy C, Oppenheim C, Mas JL. Posterior reversible encephalopathy syndrome. Handb Clin Neurol 2014;121:1687-701.

8. Sudulagunta SR, Sodalagunta MB, Kumbhat M, Settikere Nataraju A. Posterior reversible encephalopathy syndrome (PRES). Oxf Med Case Rep 2017;2017:32-5.

9. Mokri B. The Monro-Kellie hypothesis: Applications in CSF volume depletion. Neurology 2001;56:1746-8.

10. Savoiardo M, Minati L, Farina L, De Simone T, Aquino D, Mea E, et al. Spontaneous intracranial hypotension with deep brain swelling. Brain 2007;130:1884-93.

Funding: None; Conflicts of Interest: None Stated.

How to cite this article: Choudhary S, Sethi KS, Nanda S, Ganesan PK, Mehndiratta MM, Anand R, et al. Spontaneous intracranial hypotension associated with posterior reversible encephalopathy syndrome. Indian J Case Reports. 2022;8(1):12-15. 